\title{
LA FECHA DE COSECHA Y LA SÍNTESIS DE COMPUESTOS VOLATÍLES EN FRUTOS ALMACENADOS DE MANZANOS ‘GOLDEN DELICIOUS’ Y 'RED DELICIOUS'
}

\section{INFLUENCE OF HARVEST DATE ON THE SYNTHESIS OF VOLATILE COMPOUNDS IN STORED FRUITS OF APPLES ‘GOLDEN DELICIOUS’ AND 'RED DELICIOUS’}

\author{
Nora A. Salas Salazar, Francisco J. Molina Corral, David I. Berlanga Reyes, Alejandro Romo Chacón y \\ Guadalupe I. Olivas*
}

\begin{abstract}
Laboratorio de Tecnología de Alimentos de Origen Vegetal, Centro de Investigación en Alimentación y Desarrollo, Unidad Cuauhtémoc. Av. Río Conchos s/n, Parque Industrial. 31570, Cd. Cuauhtémoc, Chih., México. Tel: 01625 581-2920, Fax: 01625 581-2921.

* Autor para correspondencia (golivas@ciad.mx)
\end{abstract}

\section{RESUMEN}

Se estudió el efecto de la fecha de cosecha en la producción de compuestos volátiles en frutos de dos variedades de manzana (Malus domestica Borkh.): 'Golden Delicious', cosechados antes del inicio del climaterio (concentración interna de etileno, CIE $=0.003 \mathbf{p p m}$ ) y al inicio del climaterio (CIE $=0.9 \mathrm{ppm}$ ), a los 157 y $176 \mathrm{~d}$ después de plena floración (ddpf), respectivamente; 'Red Delicious', cosechados al inicio del climaterio $(\mathrm{CIE}=0.3 \mathrm{ppm})$ y en el climaterio $(\mathrm{CIE}=17 \mathrm{ppm})$, a los 176 y 191 ddpf, respectivamente. Los frutos de ambas variedades fueron almacenados a $0^{\circ} \mathrm{C}$ por $150 \mathrm{~d}$, y cada $30 \mathrm{~d}$ se analizó el perfil del aroma. Durante el almacenamiento la manzana 'Golden Delicious' cosechada a los $157 \mathrm{ddpf}$ presentó una mayor concentración $(\mathrm{P} \leq 0.05)$ de los hexenal, hexanal y hexanol, compuestos del sabor característicos de una manzana verde, en comparación con la cosechada a los 176 ddpf. Esta última cosecha produjo mayor concentración $(P \leq 0.05)$ de los alcoholes 1-butanol, y 2-metil-1-butanol, y del éster acetato de butilo, característicos del sabor dulce afrutado de una manzana madura. La manzana 'Red Delicious' cosechada a los 176 ddpf mostró una concentración mayor $(P \leq 0.05)$ de cis-3-hexenal, acetato de butilo y acetato 2-metil butilo importantes contribuyentes del sabor, en comparación con la cosechada a los 190 ddpf. Estos resultados muestran la estrecha asociación del estado de maduración del fruto de manzana con la producción de los compuestos volátiles del sabor durante el almacenamiento, y demuestra que la cosecha muy temprana o muy tardía puede afectar negativamente la producción de estos compuestos y el desarrollo del sabor durante el almacenamiento.

Palabra clave: Malus domestica, fecha de cosecha, sabor del fruto, almacenamiento, compuestos volátiles.

\section{SUMMARY}

The effect of harvest date on the production of volatile compounds in fruits two apple (Malus domestica Borkh.) varieties, 'Golden Delicious' and 'Red Delicious', was studied. 'Golden Delicious' fruits were harvested before the beginning of climacteric peak (internal ethylene concentration, IEC $=0.003 \mathbf{p p m}$ ) and at the beginning of climacteric peak $($ IEC $=0.9 \mathrm{ppm})$, at 157 and $176 \mathrm{~d}$ after full bloom (dafb), respectively. 'Red Delicious' fruits were harvested at the beginning of climacteric peak $($ IEC $=0.3 \mathrm{ppm})$ and at climacteric peak $(\mathrm{IEC}=17 \mathrm{ppm})$, at 176 and 191 dafb, respectively. The fruits of both varieties were stored at $0{ }^{\circ} \mathrm{C}$ during $150 \mathrm{~d}$, and the aroma profile was analyzed every $30 \mathrm{~d}$. During most of the storge time 'Golden Delicious' fruits harvested at $157 \mathrm{dafb}$ showed a higher content $(\mathrm{P} \leq \mathbf{0 . 0 5})$ of aldehydes hexenal, hexanal and hexanol, all of these flavor compounds characteristic of unripe apple during most of the storage time, as compared to the fruits harvested at the beginning of climacteric peak $(176 \mathrm{dafb})$. The later harvest produced the greatest concentration $(P \leq 0.05)$ of the alcohols 1-butanol and 2-methyl-1-butanol, and of the ester butyl acetate, characteristic compounds of the sweet fruity flavor of a ripe apple. 'Red Delicious' fruits harvested at $\mathbf{1 7 6}$ dafb showed a considerably higher amount of cis-3-hexanal, butyl acetate and 2-methyl butyl acetate, important constituents of flavor, than the those harvested at $190 \mathrm{dafb}$. These results show the relationship between apple maturity and the production of flavor volatile compounds during storage, evidencing that harvesting too early or late may affect negatively the production of these compounds and the flavor development during storage.

Index words: Malus domestica, harvest date, fruit flavor, storage, volatile compounds.

\section{INTRODUCCIÓN}

El Estado de Chihuahua es el principal productor de manzana (Malus domestica Borkh.) en la República Mexicana, con $70 \%$ de la producción nacional, donde las variedades más producidas son 'Golden Delicious' y 'Red Delicious, con 54 y $37 \%$ respectivamente (SIAP, 2009).

Las características de calidad de la manzana involucran atributos visuales como tamaño, color y frescura; y atributos no visuales como sabor y textura (Cunningham et al., 1986). Entre los atributos de calidad el sabor juega un papel central ya que propicia la aceptación del consumidor; cuando las expectativas del sabor son satisfechas, la experiencia se recordará y la compra se repetirá (Wyllie, 2008). 
Encuestas e investigaciones han coincidido en que la manzana de Chihuahua es preferida por el consumidor mexicano debido a su intenso sabor (Bismark, 2002). Los componentes del sabor se dividen en dos partes principales: el gusto y el aroma. La percepción de sustancias químicas del gusto es gracias a los receptores de sabor que hay en la lengua, que pueden reconocer los sabores dulce, ácido, salado, amargo y umami (Beaulieu y Baldwin, 2002; Yahia, 1994). Por su parte, el aroma es determinado por compuestos volátiles que tienen una importante contribución en la percepción y caracterización del sabor, ya que el aroma da el sabor característico a cada fruto (Pérez y Sanz, 2008; Baldwin, 2002).

En la manzana se han identificado alrededor de 400 compuestos volátiles (Forney et al., 2009), algunos de los cuales están presentes en mínimas concentraciones (Kays y Paull, 2004); sin embargo, sólo unos cuantos volátiles impactan de manera considerable al sabor. Los principales compuestos volátiles que definen el aroma de la manzana son ésteres, alcoholes y aldehídos, cuyas biosíntesis se llevan a cabo durante el desarrollo de la madurez del fruto mediante distintas rutas bioquímicas que involucran varias enzimas y sustratos, a partir de ácidos grasos y aminoácidos como precursores principales, los cuales son catabolizados a través de rutas como: $\beta$-oxidación, ruta de la lipooxigenasa (LOX), y ruta de los aminoácidos. Durante su maduración la manzana primeramente sintetiza aldehídos, los cuales son sustratos para la síntesis de alcoholes y estos últimos a su vez son sustratos para la síntesis de ésteres (Pérez y Sanz,
2008; Wyllie y Fellman, 2000).

Investigaciones previas han evidenciado que la manzana cosechada en diferentes estados de madurez puede variar en el perfil de compuestos volátiles durante almacenamiento (Vanoli et al., 1995; Song y Bangerth, 1996). En manzanas inmaduras predominan los aldehídos, mientras que en manzanas maduras prevalecen los ésteres, y estos últimos, son los compuestos de mayor impacto en el sabor del fruto (Salas-Salazar y Olivas, 2011).

Usualmente la elección del momento apropiado para la cosecha de la manzana se basa en el desarrollo de la firmeza, el color, el contenido de sólidos solubles, el valor de acidez titulable y el índice de almidón, más que en la optimización del aroma de la fruta (Vanoli et al., 1995), característica ésta a la que se debería prestar especial atención para lograr la satisfacción y preferencia de los consumidores. Por ello, el objetivo del presente trabajo fue estudiar el efecto de la fecha de cosecha en la producción de compuestos volátiles de las variedades de manzana 'Golden Delicious' y Red Delicious' del Estado de Chihuahua, durante su posterior almacenamiento.

\section{MATERIALES Y MÉTODOS}

\section{Material, condiciones de cosecha y almacenamiento}

Frutos de las variedades 'Golden Delicious' y 'Red Delicious' se cosecharon de árboles de 35 años de edad de

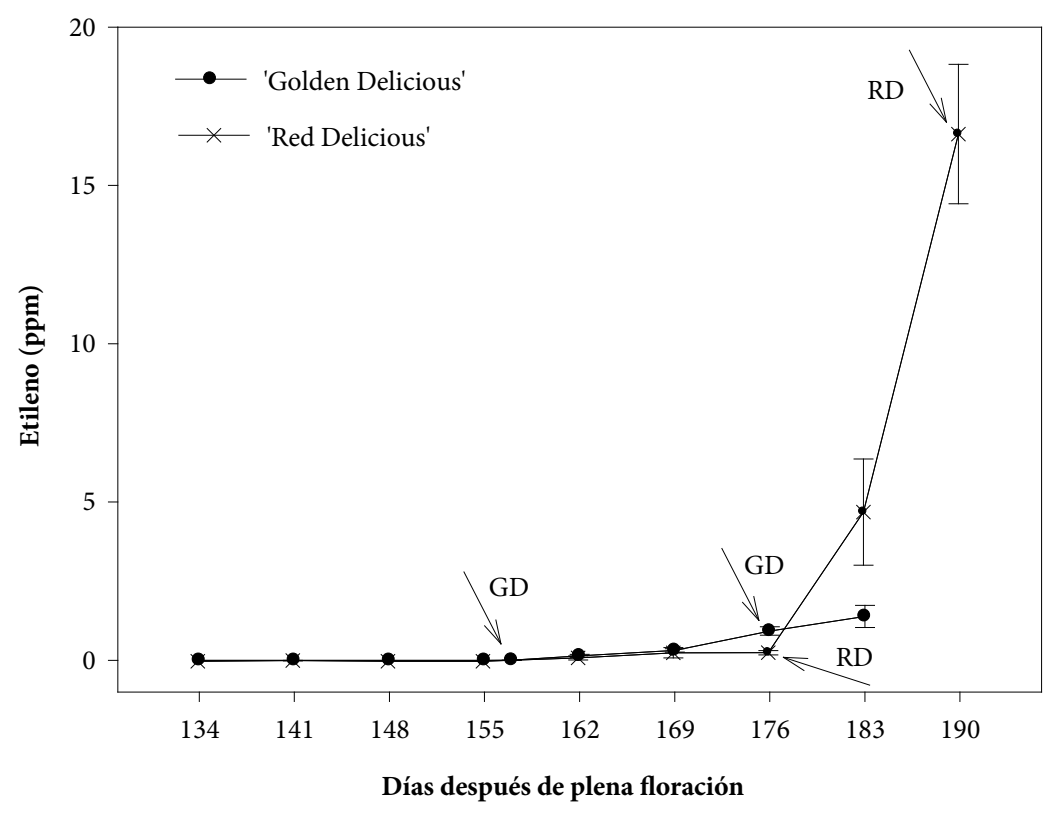

Figura 1. Evolución de la concentración interna de etileno (CIE). Las barras verticales representan el error estándar de 20 repeticiones. GD = 'Golden Delicious'; RD 'Red Delicious'. 
un huerto comercial ubicado en Cuauhtémoc, Chihuahua, México. La concentración interna de etileno fue monitoreada desde $134 \mathrm{~d}$ después de plena floración (ddpf) en ambas variedades, para definir la fecha de cosecha. Los frutos de 'Golden Delicious' fueron cosechados antes del climaterio (con concentración interna de etileno, $\mathrm{CIE}=$ $0.003 \mathrm{ppm}, 0.003 \mathrm{Mg} \mathrm{g}^{-1}$ ) y al inicio del climaterio (CIE = $0.9 \mathrm{ppm}$ ) a los 157 y $176 \mathrm{ddpf}$, respectivamente; y los de 'Red Delicious' se cosecharon al inicio del climaterio ( $\mathrm{CIE}=$ $0.3 \mathrm{ppm}$ ) y en el climaterio (CIE = $17 \mathrm{ppm})$, a los 176 y 191 ddpf respectivamente (Figura 1).

Los frutos cosechados se almacenaron a $0{ }^{\circ} \mathrm{C}$ durante 5 meses en cámaras de refrigeración separadas (una por variedad), y luego evaluados periódicamente por su composición y concentración de compuestos volátiles.

\section{Concentración interna de etileno (CIE)}

La CIE de los frutos se evaluó semanalmente a partir de 134 ddpf y hasta la cosecha. La extracción se llevó a cabo con una jeringa de $1 \mathrm{~mL}$ provista con aguja hipodérmica que se introdujo a través del cáliz de la fruta para extraer un volumen de $0.2 \mathrm{~mL}$ del espacio interior; en seguida, la aguja se insertó en un tapón de goma para sellarla. Previo a la obtención de la muestra, los frutos se sumergieron en agua para evitar la contaminación con aire atmosférico (Saltveilt, 1982). Las muestras de gas del cáliz se analizaron en un cromatógrafo de gases (Varian $3800 \AA$, USA) equipado con una columna empacada Hayesep Q Chromapack ${ }^{\circledR}$ de $1.8 \mathrm{~m}$ de largo y con un detector de ionización de flama (FID); se usó helio como gas acarreador a un flujo de 30 $\mathrm{mL} \mathrm{min}^{-1}$. Las condiciones cromatográficas fueron las siguientes: temperatura inicial del horno $60^{\circ} \mathrm{C}$ durante 2 min, que se incrementó hasta $120^{\circ} \mathrm{C}$ a razón de $60{ }^{\circ} \mathrm{C}$ $\mathrm{min}^{-1}$, y esta temperatura final se mantuvo por $1.5 \mathrm{~min}$. El promedio de CIE se calculó del análisis de 20 manzanas por variedad.

\section{Análisis de compuestos volátiles}

La concentración de compuestos volátiles en los frutos de manzana se determinó por cromatografía de gases acoplada a espectrometría de masas (Varian Saturn $2100 D \circledR$ GC/MS), con la técnica de microextracción en fase sólida (SPME). Se utilizó un procesador de alimentos (Turmix ${ }^{\circledR}$ ) para obtener jugo de manzana, en ocho manzanas por repetición; el jugo se colocó en un vial de $20 \mathrm{~mL}$, se congeló en nitrógeno líquido y se almacenó a $-70{ }^{\circ} \mathrm{C}$ para su posterior análisis. Las muestras de jugo se descongelaron en baño agitado a $25{ }^{\circ} \mathrm{C}$ y se colocaron $2 \mathrm{~mL}$ en viales de $4 \mathrm{~mL}$ que contenían $0.65 \mathrm{~g}$ de $\mathrm{NaCl}$ y una barra magnética para su agitación. Para la adsorción de los compuestos volátiles del espacio de cabeza del vial se utilizó una fibra PDMS-DVB® (65 $\mu \mathrm{m}$, Supelco), que se expuso durante $60 \mathrm{~min}$ a temperatura ambiente con agitación.

Los compuestos volátiles fueron posteriormente desorbidos en el puerto de inyección del cromatógrafo de gases equipado con una columna capilar Equity-1 ${ }^{\circledR}(60$ $\mathrm{m} \times 0.25 \mathrm{~mm} \times 0.25 \mu \mathrm{m}$; Supelco, USA). Las condiciones cromatográficas fueron las siguientes: temperatura del inyector $200{ }^{\circ} \mathrm{C}$, temperatura inicial del horno $33{ }^{\circ} \mathrm{C}$ sostenida durante $5 \mathrm{~min}$, que se incrementó hasta $50{ }^{\circ} \mathrm{C}$ a razón de $2{ }^{\circ} \mathrm{C} \mathrm{min}{ }^{-1}$, y luego hasta $250{ }^{\circ} \mathrm{C}$ a razón de 5 ${ }^{\circ} \mathrm{C} \min ^{-1}$, y esta última se mantuvo durante $6.5 \mathrm{~min}$. Se utilizó helio como gas acarreador, a una velocidad lineal de $30 \mathrm{~cm} \mathrm{~s}^{-1}$. Los espectros de masas se obtuvieron mediante ionización electrónica a 70 eV. Las temperaturas de la línea de transferencia y de la trampa de iones fueron 250 y 180 ${ }^{\circ} \mathrm{C}$, respectivamente. La identificación de los compuestos volátiles se hizo por comparación de los espectros de masas obtenidos de las muestras contra los reportados en la biblioteca NIST 98, y contra los espectros obtenidos a partir de estándares de alta pureza (Sigma-Aldrich y ChemService) analizados bajo idénticas condiciones que las muestras. Todos los valores reportados representan el promedio de tres repeticiones.

\section{Análisis estadístico}

Para el análisis estadístico de los datos se utilizó un diseño completamente al azar con parcelas divididas en el tiempo, mediante el programa de computadora SAS Versión 9 (SAS Institute, 2010).

\section{RESULTADOS Y DISCUSIÓN}

Los principales compuestos volátiles (aldehídos, alcoholes y ésteres) emitidos por los frutos de manzana de 'Golden Delicious' y de 'Red Delicious' cosechados en dos estados de madurez y almacenados en refrigeración, se muestran en los Cuadros 1 al 6.

Los compuestos volátiles que se encontraron en mayor concentración en frutos de 'Golden Delicious' al momento de cosecha en las dos fechas fueron los aldehídos (Figura 2), compuestos que son los primeros en ser sintetizados en las rutas metabólicas de la producción del sabor, que son predominantes en manzanas inmaduras (De Pooter et al., 1987) y aportan el sabor a "fruta verde" (Mattheis et al., 1991), como ocurre sobre todo en frutos cosechados tempranamente (157 ddpf). También los frutos de 'Red Delicious' cosechados en la primera fecha de cosecha (176 ddpf) tuvieron mayor concentración de aldehídos ( $\mathrm{P} \leq$ 0.05) que los cosechados $14 \mathrm{~d}$ después (191 ddpf) (Figura $3)$. 
Cuadro 1. Concentración de aldehídos volátiles (ppm) en frutos de la manzana ‘Golden Delicious' cosechados en dos estados de madurez ( 157 y 176 días después de plena floración, ddpf).

\begin{tabular}{llllllll}
\hline \multirow{2}{*}{ Aldehídos } & & \multicolumn{7}{c}{ Almacenamiento (días) } \\
\cline { 3 - 8 } & ddpf & 0 (cosecha) & 30 & 60 & 90 & 120 & 150 \\
\hline \multirow{3}{*}{ Butanal } & & & & & & & \\
& 157 & $0.0007 \mathrm{a}$ & $0.012 \mathrm{a}$ & $0.338 \mathrm{a}$ & $0.463 \mathrm{a}$ & $0.755 \mathrm{a}$ & $0.871 \mathrm{a}$ \\
& 176 & $\operatorname{Tr}$ & $0.068 \mathrm{a}$ & $0.472 \mathrm{a}$ & $0.217 \mathrm{~b}$ & $0.161 \mathrm{~b}$ & $0.080 \mathrm{~b}$ \\
Petil butanal & 157 & $\mathrm{ND}$ & $\operatorname{Tr}$ & $\operatorname{Tr}$ & $0.001 \mathrm{a}$ & $0.001 \mathrm{a}$ & $0.001 \mathrm{a}$ \\
& 176 & $\mathrm{ND}$ & 0.002 & 0.002 & $0.005 \mathrm{~b}$ & $0.003 \mathrm{~b}$ & $0.001 \mathrm{a}$ \\
cis 3-hexenal & 157 & $0.002 \mathrm{a}$ & $0.001 \mathrm{a}$ & $0.001 \mathrm{a}$ & $0.001 \mathrm{a}$ & $0.001 \mathrm{a}$ & $0.002 \mathrm{a}$ \\
& 176 & $\operatorname{Tr}$ & $0.002 \mathrm{a}$ & $0.002 \mathrm{a}$ & $0.002 \mathrm{a}$ & $0.002 \mathrm{a}$ & $0.001 \mathrm{a}$ \\
Hexanal & 157 & $15.327 \mathrm{a}$ & $0.023 \mathrm{a}$ & $0.152 \mathrm{a}$ & $0.108 \mathrm{a}$ & $0.076 \mathrm{a}$ & $0.026 \mathrm{a}$ \\
& 176 & $0.154 \mathrm{~b}$ & $0.096 \mathrm{a}$ & $0.110 \mathrm{a}$ & $0.098 \mathrm{a}$ & $0.097 \mathrm{a}$ & $0.149 \mathrm{a}$ \\
2 Hexenal & 157 & $5.918 \mathrm{a}$ & $3.342 \mathrm{a}$ & $4.640 \mathrm{a}$ & $18.336 \mathrm{a}$ & $105.278 \mathrm{a}$ & $68.395 \mathrm{a}$ \\
& 176 & $1.577 \mathrm{a}$ & $6.032 \mathrm{a}$ & $9.419 \mathrm{a}$ & $15.740 \mathrm{a}$ & $19.314 \mathrm{~b}$ & $18.164 \mathrm{~b}$ \\
Benzaldehínyyyyyy & 157 & $60.890 \mathrm{a}$ & $18.947 \mathrm{a}$ & $32.215 \mathrm{a}$ & $39.695 \mathrm{a}$ & $50.810 \mathrm{a}$ & $26.133 \mathrm{a}$ \\
\multirow{2}{*}{ Nonanal } & 176 & $3.640 \mathrm{~b}$ & $4.571 \mathrm{~b}$ & $16.923 \mathrm{~b}$ & $12.427 \mathrm{~b}$ & $11.944 \mathrm{~b}$ & $3.922 \mathrm{~b}$ \\
& 157 & 0.0006 & $\operatorname{Tr}$ & $\operatorname{Tr}$ & $\operatorname{Tr}$ & 0.001 & $0.0010 \mathrm{a}$ \\
Decanal & 176 & $\operatorname{Tr}$ & $\operatorname{Tr}$ & $\operatorname{Tr}$ & $\operatorname{Tr}$ & $\operatorname{Tr}$ & $0.0008 \mathrm{a}$ \\
& 157 & $0.002 \mathrm{a}$ & $0.001 \mathrm{a}$ & $0.002 \mathrm{a}$ & $0.002 \mathrm{a}$ & $0.003 \mathrm{a}$ & $0.003 \mathrm{a}$ \\
& 176 & $0.0007 \mathrm{a}$ & $0.001 \mathrm{a}$ & $0.002 \mathrm{a}$ & $0.002 \mathrm{a}$ & $0.002 \mathrm{a}$ & $0.002 \mathrm{a}$
\end{tabular}

Medias entre fechas de cosecha dentro de un mismo compuesto y seguidas por la misma letra minúscula, no son significativamente diferentes (Tukey, 0.05). $\mathrm{Tr}=$ Trazas, $<0.0005 \mathrm{ppm} ; \mathrm{ND}=$ compuestos no detectados.

Cuadro 2. Concentración de alcoholes volátiles (ppm) en frutos de la manzana 'Golden Delicious' cosechados en dos estados de madurez ( 157 y 176 días después de plena floración, ddpf).

\begin{tabular}{llllllll}
\hline \multirow{2}{*}{ Alcoholes } & & \multicolumn{7}{c}{ Almacenamiento (días) } \\
\cline { 3 - 7 } 1 Butanol & ddpf & 0 (cosecha) & 30 & 60 & 90 & 120 & 150 \\
& & & & & & & \\
2 Metil 1 propanol & 157 & $\operatorname{Tr}$ & $\operatorname{Tr}$ & $0.690 \mathrm{a}$ & $3.532 \mathrm{a}$ & $0.822 \mathrm{a}$ & $3.471 \mathrm{a}$ \\
& 176 & 0.033 & 3.822 & $2.895 \mathrm{~b}$ & $7.541 \mathrm{~b}$ & $0.902 \mathrm{a}$ & $1.227 \mathrm{~b}$ \\
2 Metil 1 Butanol & 157 & $0.136 \mathrm{a}$ & $0.294 \mathrm{a}$ & $0.359 \mathrm{a}$ & $0.383 \mathrm{a}$ & $1.521 \mathrm{a}$ & $0.729 \mathrm{a}$ \\
& 176 & $0.448 \mathrm{~b}$ & $0.455 \mathrm{a}$ & $0.671 \mathrm{~b}$ & $0.674 \mathrm{~b}$ & $0.858 \mathrm{~b}$ & $0.542 \mathrm{~b}$ \\
1 Pentanol & 157 & $0.577 \mathrm{a}$ & $1.128 \mathrm{a}$ & $1.525 \mathrm{a}$ & $2.338 \mathrm{a}$ & $3.705 \mathrm{a}$ & $13.461 \mathrm{a}$ \\
& 176 & $2.525 \mathrm{a}$ & $2.170 \mathrm{a}$ & $4.143 \mathrm{a}$ & $31.314 \mathrm{~b}$ & $24.355 \mathrm{~b}$ & $20.165 \mathrm{a}$ \\
3-Hexen-1-ol (Z) & 157 & $0.007 \mathrm{a}$ & $0.002 \mathrm{a}$ & $0.006 \mathrm{a}$ & $0.008 \mathrm{a}$ & $0.008 \mathrm{a}$ & $0.010 \mathrm{a}$ \\
& 176 & $0.012 \mathrm{a}$ & $0.011 \mathrm{a}$ & $0.012 \mathrm{a}$ & $0.014 \mathrm{a}$ & $0.002 \mathrm{a}$ & $0.003 \mathrm{a}$ \\
2-Hexen-1-ol (E)- & 157 & $0.075 \mathrm{a}$ & $\operatorname{Tr}$ & $\operatorname{Tr}$ & $\operatorname{Tr}$ & $\operatorname{Tr}$ & $\operatorname{Tr}$ \\
& 176 & $0.003 \mathrm{~b}$ & $\operatorname{Tr}$ & $\operatorname{Tr}$ & $\operatorname{Tr}$ & $\operatorname{Tr}$ & $\operatorname{Tr}$ \\
1-Hexanol & 157 & $0.011 \mathrm{a}$ & $\operatorname{Tr}$ & $\operatorname{Tr}$ & $\operatorname{Tr}$ & $\operatorname{Tr}$ & $\operatorname{Tr}$ \\
& 176 & $0.005 \mathrm{~b}$ & $\operatorname{Tr}$ & $\operatorname{Tr}$ & $\operatorname{Tr}$ & $\operatorname{Tr}$ & 0.0006 \\
1-Heptanol & 157 & $0.061 \mathrm{a}$ & $32.482 \mathrm{a}$ & $32.957 \mathrm{a}$ & $36.099 \mathrm{a}$ & $32.806 \mathrm{a}$ & $33.652 \mathrm{a}$ \\
& 176 & $0.060 \mathrm{a}$ & $4.781 \mathrm{~b}$ & $13.243 \mathrm{~b}$ & $2.303 \mathrm{~b}$ & $1.446 \mathrm{~b}$ & $0.348 \mathrm{~b}$ \\
2 Etil 1 hexanol & 157 & $0.002 \mathrm{a}$ & $0.001 \mathrm{a}$ & $0.002 \mathrm{a}$ & $0.002 \mathrm{a}$ & $0.003 \mathrm{a}$ & $0.005 \mathrm{a}$ \\
& 176 & $0.003 \mathrm{~b}$ & $0.002 \mathrm{~b}$ & $0.002 \mathrm{a}$ & $0.004 \mathrm{~b}$ & $0.003 \mathrm{a}$ & $0.003 \mathrm{~b}$ \\
1-Octanol & 157 & $0.0008 \mathrm{a}$ & $\operatorname{Tr}$ & $\operatorname{Tr}$ & $\operatorname{Tr}$ & $\operatorname{Tr}$ & $0.001 \mathrm{a}$ \\
& 176 & $0.0005 \mathrm{a}$ & 0.0005 & 0.0005 & 0.0009 & 0.001 & $0.001 \mathrm{a}$ \\
& 157 & $0.0005 \mathrm{a}$ & $0.0007 \mathrm{a}$ & $\operatorname{Tr}$ & $0.0008 \mathrm{a}$ & $\operatorname{Tr}$ & $0.001 \mathrm{a}$ \\
& 176 & $0.001 \mathrm{~b}$ & $0.0006 \mathrm{a}$ & $\operatorname{Tr}$ & $0.0006 \mathrm{a}$ & 0.0005 & $0.0008 \mathrm{a}$ \\
\hline
\end{tabular}

Medias entre fechas de cosecha, dentro de un mismo compuesto y seguidas por la misma letra minúscula, no son significativamente diferentes (Tukey, 0.05). $\mathrm{Tr}=$ Trazas, $<0.0005 \mathrm{ppm} ; \mathrm{ND}=$ compuestos no detectados. 
Cuadro 3. Concentración de ésteres volátiles (ppm) en frutos de la manzana 'Golden Delicious' cosechados en dos estados de madurez (157 y 176 días después de plena floración, ddpf).

\begin{tabular}{|c|c|c|c|c|c|c|c|}
\hline \multirow{2}{*}{ Ésteres } & \multicolumn{7}{|c|}{ Almacenamiento (días) } \\
\hline & ddpf & 0 (cosecha) & 30 & 60 & 90 & 120 & 150 \\
\hline \multirow[t]{2}{*}{ Acetato de etilo } & 157 & $0.003 \mathrm{a}$ & $0.003 \mathrm{a}$ & $0.005 \mathrm{a}$ & $0.005 \mathrm{a}$ & $0.007 \mathrm{a}$ & $0.008 \mathrm{a}$ \\
\hline & 176 & $0.002 \mathrm{a}$ & $0.006 \mathrm{~b}$ & $0.006 \mathrm{a}$ & $0.013 \mathrm{~b}$ & $0.011 \mathrm{~b}$ & $0.012 \mathrm{~b}$ \\
\hline \multirow[t]{2}{*}{ Propionato de etilo } & 157 & $\mathrm{ND}$ & ND & ND & ND & ND & ND \\
\hline & 176 & ND & ND & ND & $\operatorname{Tr}$ & $\operatorname{Tr}$ & 0.353 \\
\hline \multirow[t]{2}{*}{ Acetato de propilo } & 157 & $\operatorname{Tr}$ & $0.0009 \mathrm{a}$ & $0.017 \mathrm{a}$ & $0.022 \mathrm{a}$ & $0.307 \mathrm{a}$ & $0.125 \mathrm{a}$ \\
\hline & 176 & 0.002 & $0.002 \mathrm{a}$ & $0.002 \mathrm{a}$ & $0.124 \mathrm{~b}$ & $0.265 \mathrm{a}$ & $0.151 \mathrm{a}$ \\
\hline \multirow[t]{2}{*}{ Butanoato de metilo } & 157 & ND & ND & ND & ND & ND & ND \\
\hline & 176 & ND & ND & ND & ND & ND & 0.002 \\
\hline \multirow[t]{2}{*}{ Acetato de 2 metil propilo } & 157 & $0.0007 \mathrm{a}$ & $0.001 \mathrm{a}$ & $0.006 \mathrm{a}$ & $0.013 \mathrm{a}$ & $0.027 \mathrm{a}$ & $0.024 \mathrm{a}$ \\
\hline & 176 & $0.006 \mathrm{a}$ & $0.011 \mathrm{a}$ & $0.039 \mathrm{~b}$ & $0.009 \mathrm{a}$ & $0.024 \mathrm{a}$ & $0.015 \mathrm{a}$ \\
\hline \multirow[t]{2}{*}{ Metil 2 metil butanoato } & 157 & $\mathrm{ND}$ & ND & ND & ND & ND & ND \\
\hline & 176 & ND & ND & ND & ND & ND & ND \\
\hline \multirow[t]{2}{*}{ Butanoato de etilo } & 157 & ND & ND & ND & ND & ND & $\mathrm{ND}$ \\
\hline & 176 & ND & ND & ND & ND & ND & $0.009 \mathrm{a}$ \\
\hline \multirow[t]{2}{*}{ Propanoato de propilo } & 157 & $\operatorname{Tr}$ & $\operatorname{Tr}$ & $\operatorname{Tr}$ & $\operatorname{Tr}$ & $\operatorname{Tr}$ & $\operatorname{Tr}$ \\
\hline & 176 & $\operatorname{Tr}$ & $\operatorname{Tr}$ & $\operatorname{Tr}$ & $\operatorname{Tr}$ & $\operatorname{Tr}$ & 0.007 \\
\hline \multirow[t]{2}{*}{ Acetato de butilo } & 157 & $0.027 \mathrm{a}$ & $28.581 \mathrm{a}$ & $42.066 \mathrm{a}$ & $46.640 \mathrm{a}$ & $44.398 \mathrm{a}$ & $18.415 \mathrm{a}$ \\
\hline & 176 & $0.774 \mathrm{a}$ & $46.938 \mathrm{~b}$ & $62.540 \mathrm{~b}$ & $68.420 \mathrm{~b}$ & $35.446 \mathrm{a}$ & $5.908 \mathrm{a}$ \\
\hline \multirow[t]{2}{*}{ 2-Metil butanoato de etilo } & 157 & ND & $\mathrm{ND}$ & $\mathrm{ND}$ & $\mathrm{ND}$ & $\mathrm{ND}$ & $\operatorname{Tr}$ \\
\hline & 176 & ND & ND & ND & ND & ND & 0.014 \\
\hline \multirow[t]{2}{*}{ Acetato de 2 metil butilo } & 157 & $0.015 \mathrm{a}$ & $0.020 \mathrm{a}$ & $0.396 \mathrm{a}$ & $1.147 \mathrm{a}$ & $1.018 \mathrm{a}$ & $1.016 \mathrm{a}$ \\
\hline & 176 & $0.236 \mathrm{a}$ & $0.487 \mathrm{~b}$ & $0.519 \mathrm{a}$ & $1.298 \mathrm{a}$ & $0.613 \mathrm{~b}$ & $0.570 \mathrm{~b}$ \\
\hline \multirow[t]{2}{*}{ Pentanoato de etilo } & 157 & ND & $\operatorname{Tr}$ & $0.001 \mathrm{a}$ & $0.003 \mathrm{a}$ & $0.002 \mathrm{a}$ & $0.003 \mathrm{a}$ \\
\hline & 176 & $\operatorname{Tr}$ & 0.001 & $0.001 \mathrm{a}$ & $0.003 \mathrm{a}$ & $0.003 \mathrm{a}$ & $0.002 \mathrm{a}$ \\
\hline \multirow[t]{2}{*}{ Propanoato de butilo } & 157 & $\operatorname{Tr}$ & $0.002 \mathrm{a}$ & $0.017 \mathrm{a}$ & $0.013 \mathrm{a}$ & $0.009 \mathrm{a}$ & $0.007 \mathrm{a}$ \\
\hline & 176 & 0.002 & $0.004 \mathrm{a}$ & $0.005 \mathrm{~b}$ & $0.015 \mathrm{a}$ & $0.013 \mathrm{a}$ & $0.013 \mathrm{~b}$ \\
\hline \multirow[t]{2}{*}{ Acetato de pentilo } & 157 & $0.005 \mathrm{a}$ & $0.021 \mathrm{a}$ & $0.029 \mathrm{a}$ & $0.029 \mathrm{a}$ & $0.028 \mathrm{a}$ & $0.027 \mathrm{a}$ \\
\hline & 176 & $0.007 \mathrm{a}$ & $0.023 \mathrm{a}$ & $0.019 \mathrm{a}$ & $0.021 \mathrm{a}$ & $0.026 \mathrm{a}$ & $0.029 \mathrm{a}$ \\
\hline \multirow[t]{2}{*}{ Acetato de 3 metil buten-1-ol } & 157 & ND & ND & ND & ND & ND & $\mathrm{ND}$ \\
\hline & 176 & 0.001 & $\mathrm{NDl}$ & $\mathrm{ND}$ & ND & $\mathrm{ND}$ & $\mathrm{ND}$ \\
\hline \multirow[t]{2}{*}{ Butanoato de butilo } & 157 & $\operatorname{Tr}$ & $0.020 \mathrm{a}$ & $0.032 \mathrm{a}$ & $0.058 \mathrm{a}$ & $0.052 \mathrm{a}$ & $0.067 \mathrm{a}$ \\
\hline & 176 & 0.002 & $0.029 \mathrm{a}$ & $0.058 \mathrm{a}$ & $0.051 \mathrm{a}$ & $0.046 \mathrm{a}$ & $0.035 \mathrm{~b}$ \\
\hline \multirow[t]{2}{*}{ Acetato de cis-3-hexenilo } & 157 & $0.002 \mathrm{a}$ & $\operatorname{Tr}$ & $\operatorname{Tr}$ & $\operatorname{Tr}$ & $\operatorname{Tr}$ & $0.0005 \mathrm{a}$ \\
\hline & 176 & $0.0008 \mathrm{~b}$ & $\operatorname{Tr}$ & $\operatorname{Tr}$ & $\operatorname{Tr}$ & $\operatorname{Tr}$ & $\operatorname{Tr}$ \\
\hline Acetato de hexilo & 157 & $0.018 \mathrm{a}$ & $0.954 \mathrm{a}$ & $0.925 \mathrm{a}$ & $1.276 \mathrm{a}$ & $1.385 \mathrm{a}$ & $1.627 \mathrm{a}$ \\
\hline & 176 & $0.034 \mathrm{a}$ & $0.826 \mathrm{a}$ & $0.807 \mathrm{a}$ & $0.838 \mathrm{a}$ & $0.832 \mathrm{a}$ & $0.958 \mathrm{~b}$ \\
\hline 2-Metil butanoato de butilo & 157 & $\operatorname{Tr}$ & $0.0005 \mathrm{a}$ & $0.003 \mathrm{a}$ & $0.003 \mathrm{a}$ & $0.001 \mathrm{a}$ & $0.002 \mathrm{a}$ \\
\hline & 176 & 0.0005 & $0.0005 \mathrm{a}$ & $\operatorname{Tr}$ & $0.002 \mathrm{a}$ & $0.003 \mathrm{a}$ & $0.002 \mathrm{a}$ \\
\hline Hexanoato de propilo & 157 & $0.001 \mathrm{a}$ & $0.001 \mathrm{a}$ & $0.001 \mathrm{a}$ & $0.002 \mathrm{a}$ & $0.002 \mathrm{a}$ & $0.002 \mathrm{a}$ \\
\hline & 176 & $0.001 \mathrm{a}$ & $0.001 \mathrm{a}$ & $0.001 \mathrm{a}$ & $0.001 \mathrm{a}$ & $0.002 \mathrm{a}$ & $0.002 \mathrm{a}$ \\
\hline Propanoato de hexilo & 157 & $\operatorname{Tr}$ & $0.001 \mathrm{a}$ & $0.001 \mathrm{a}$ & $0.001 \mathrm{a}$ & $0.002 \mathrm{a}$ & $0.002 \mathrm{a}$ \\
\hline & 176 & 0.0005 & $0.001 \mathrm{a}$ & $0.001 \mathrm{a}$ & $0.001 \mathrm{a}$ & $0.002 \mathrm{a}$ & $0.003 \mathrm{a}$ \\
\hline Butanoato de hexilo & 157 & $\operatorname{Tr}$ & $0.008 \mathrm{a}$ & $0.016 \mathrm{a}$ & $0.016 \mathrm{a}$ & $0.014 \mathrm{a}$ & $0.012 \mathrm{a}$ \\
\hline & 176 & $\operatorname{Tr}$ & $0.011 \mathrm{a}$ & $0.016 \mathrm{a}$ & $0.014 \mathrm{a}$ & $0.011 \mathrm{a}$ & $0.010 \mathrm{a}$ \\
\hline Octanoato de etilo & 157 & $0.001 \mathrm{a}$ & $0.001 \mathrm{a}$ & $0.003 \mathrm{a}$ & $0.003 \mathrm{a}$ & $0.003 \mathrm{a}$ & $0.003 \mathrm{a}$ \\
\hline & 176 & $0.003 \mathrm{a}$ & $0.004 \mathrm{a}$ & $0.004 \mathrm{a}$ & $0.005 \mathrm{a}$ & $0.007 \mathrm{a}$ & $0.010 \mathrm{~b}$ \\
\hline 2-Metil butanoato de hexilo & 157 & $\operatorname{Tr}$ & $0.001 \mathrm{a}$ & $0.001 \mathrm{a}$ & $0.002 \mathrm{a}$ & $0.003 \mathrm{a}$ & $0.002 \mathrm{a}$ \\
\hline & 176 & $\operatorname{Tr}$ & $0.0007 \mathrm{a}$ & $0.005 \mathrm{~b}$ & $0.002 \mathrm{a}$ & $0.001 \mathrm{a}$ & $0.002 \mathrm{a}$ \\
\hline Hexanoato de hexilo & 157 & $\operatorname{Tr}$ & $0.0007 \mathrm{a}$ & $0.004 \mathrm{a}$ & $0.003 \mathrm{a}$ & $0.010 \mathrm{a}$ & $0.005 \mathrm{a}$ \\
\hline & 176 & $\operatorname{Tr}$ & $0.002 \mathrm{~b}$ & $0.002 \mathrm{~b}$ & $0.003 \mathrm{a}$ & $0.002 \mathrm{~b}$ & $0.002 \mathrm{~b}$ \\
\hline
\end{tabular}

Medias entre fechas de cosecha dentro de un mismo compuesto y seguidas por la misma letra minúscula, no son significativamente diferentes (Tukey, 0.05). $\operatorname{Tr}=$ Trazas, $<0.0005$ ppm; $\mathrm{ND}=$ compuestos no detectados. 
Cuadro 4. Concentración de aldehídos volátiles (ppm) en frutos de la manzana 'Red Delicious' cosechados en dos estados de madurez ( 176 y 191 días después de plena floración, ddpf).

\begin{tabular}{llllllll}
\hline \multirow{2}{*}{ Aldehídos } & & \multicolumn{7}{c}{ Almacenamiento (días) } \\
\cline { 5 - 8 } Butanal & ddpf & 0 (cosecha) & 30 & 60 & 90 & 120 & 150 \\
& & & & & & & \\
2 Metil butanal & 176 & 0.002 & $0.402 \mathrm{a}$ & $0.301 \mathrm{a}$ & $0.299 \mathrm{a}$ & $0.358 \mathrm{a}$ & 0.496 \\
& 191 & $\operatorname{Tr}$ & $0.183 \mathrm{~b}$ & $0.382 \mathrm{a}$ & $0.414 \mathrm{a}$ & $0.173 \mathrm{~b}$ & $\operatorname{Tr}$ \\
Pentanal & 176 & $0.003 \mathrm{a}$ & $0.006 \mathrm{a}$ & $0.005 \mathrm{a}$ & $0.005 \mathrm{a}$ & 0.005 & 0.005 \\
& 191 & $0.003 \mathrm{a}$ & $0.006 \mathrm{a}$ & $0.003 \mathrm{a}$ & $0.003 \mathrm{a}$ & $\mathrm{ND}$ & $\mathrm{ND}$ \\
cis 3-hexenal & 176 & $\operatorname{Tr}$ & $\operatorname{Tr}$ & $\operatorname{Tr}$ & $\operatorname{Tr}$ & $\operatorname{Tr}$ & $\operatorname{Tr}$ \\
& 191 & $\operatorname{Tr}$ & $\operatorname{Tr} 1$ & $\operatorname{Tr}$ & $\operatorname{Tr}$ & $\mathrm{ND}$ & $\mathrm{ND}$ \\
Hexanal & 176 & $12.498 \mathrm{a}$ & $9.658 \mathrm{a}$ & $3.331 \mathrm{a}$ & $4.467 \mathrm{a}$ & $2.287 \mathrm{a}$ & $1.785 \mathrm{a}$ \\
& 191 & $9.214 \mathrm{~b}$ & $0.764 \mathrm{~b}$ & $0.980 \mathrm{~b}$ & $0.389 \mathrm{~b}$ & $0.594 \mathrm{~b}$ & $0.124 \mathrm{~b}$ \\
2 Hexenal & 176 & $2.041 \mathrm{a}$ & $1.462 \mathrm{a}$ & $2.674 \mathrm{a}$ & $2.460 \mathrm{a}$ & $2.775 \mathrm{a}$ & $2.721 \mathrm{a}$ \\
& 191 & $2.554 \mathrm{a}$ & $2.365 \mathrm{a}$ & $3.212 \mathrm{a}$ & $3.397 \mathrm{a}$ & $3.614 \mathrm{a}$ & $3.185 \mathrm{a}$ \\
Benzaldehído & 176 & $29.425 \mathrm{a}$ & $14.046 \mathrm{a}$ & $15.511 \mathrm{a}$ & $15.166 \mathrm{a}$ & $18.585 \mathrm{a}$ & $20.867 \mathrm{a}$ \\
& 191 & $22.555 \mathrm{~b}$ & $18.474 \mathrm{a}$ & $19.023 \mathrm{a}$ & $18.417 \mathrm{a}$ & $16.244 \mathrm{a}$ & $16.098 \mathrm{a}$ \\
Nonanal & 176 & $\operatorname{Tr}$ & $\operatorname{Tr}$ & $\operatorname{Tr}$ & $\operatorname{Tr}$ & $\operatorname{Tr}$ & 0.0009 \\
& 191 & $\operatorname{Tr}$ & $\operatorname{Tr}$ & $\operatorname{Tr}$ & $\operatorname{Tr}$ & $\operatorname{Tr}$ & $\operatorname{Tr}$ \\
Decanal & 176 & $0.002 \mathrm{a}$ & $0.001 \mathrm{a}$ & $0.002 \mathrm{a}$ & $0.002 \mathrm{a}$ & $0.003 \mathrm{a}$ & $0.002 \mathrm{a}$ \\
& 191 & $0.001 \mathrm{a}$ & $0.002 \mathrm{a}$ & $0.002 \mathrm{a}$ & $0.002 \mathrm{a}$ & $0.003 \mathrm{a}$ & $0.001 \mathrm{a}$ \\
& 176 & $\operatorname{Tr}$ & $\operatorname{Tr}$ & $\operatorname{Tr}$ & 0.001 & 0.003 & 0.002
\end{tabular}

Medias entre fechas de cosecha dentro de un mismo compuesto y seguidas por la misma letra minúscula, no son significativamente diferentes (Tukey, 0.05). Tr $=$ Trazas, $<0.0005 \mathrm{ppm}$ Trazas, $<0.0005 \mathrm{ppm}$; ND = compuestos no detectados.

Cuadro 5. Concentración de alcoholes volátiles (ppm) en frutos de la manzana 'Red Delicious' cosechados en dos estados de madurez (176 y 191 días después de plena floración, ddpf).

\begin{tabular}{|c|c|c|c|c|c|c|c|}
\hline \multirow{2}{*}{ Alcoholes } & \multicolumn{7}{|c|}{ Almacenamiento (días) } \\
\hline & ddpf & 0 (cosecha) & 30 & 60 & 90 & 120 & 150 \\
\hline \multirow[t]{2}{*}{1 Butanol } & 176 & $\operatorname{Tr}$ & $0.680 \mathrm{a}$ & $0.824 \mathrm{a}$ & $1.167 \mathrm{a}$ & $1.268 \mathrm{a}$ & $1.226 \mathrm{a}$ \\
\hline & 191 & $\operatorname{Tr}$ & $\operatorname{Tr}$ & $0.199 \mathrm{~b}$ & $0.222 \mathrm{~b}$ & $0.061 \mathrm{~b}$ & $0.176 \mathrm{~b}$ \\
\hline \multirow[t]{2}{*}{2 Metil 1 propanol } & 176 & $0.319 \mathrm{a}$ & $0.501 \mathrm{a}$ & $0.541 \mathrm{a}$ & $0.515 \mathrm{a}$ & $0.503 \mathrm{a}$ & $0.599 \mathrm{a}$ \\
\hline & 191 & $0.788 \mathrm{a}$ & $0.589 \mathrm{a}$ & $1.220 \mathrm{~b}$ & $1.176 \mathrm{~b}$ & $1.093 \mathrm{~b}$ & $2.192 \mathrm{~b}$ \\
\hline \multirow[t]{2}{*}{2 Metil 1 Butanol } & 176 & $11.119 \mathrm{a}$ & $38.185 \mathrm{a}$ & $39.938 \mathrm{a}$ & $40.189 \mathrm{a}$ & $43.873 \mathrm{a}$ & $49.684 \mathrm{a}$ \\
\hline & 191 & $30.477 \mathrm{~b}$ & $63.143 \mathrm{~b}$ & $40.501 \mathrm{a}$ & $24.453 \mathrm{a}$ & $42.983 \mathrm{a}$ & $44.023 \mathrm{a}$ \\
\hline \multirow[t]{2}{*}{1 Pentanol } & 176 & $0.005 \mathrm{a}$ & $0.006 \mathrm{a}$ & $0.007 \mathrm{a}$ & $0.009 \mathrm{a}$ & $0.008 \mathrm{a}$ & $0.009 \mathrm{a}$ \\
\hline & 191 & $0.003 \mathrm{a}$ & $0.006 \mathrm{a}$ & $0.006 \mathrm{a}$ & $0.005 \mathrm{~b}$ & $0.006 \mathrm{a}$ & $0.005 \mathrm{~b}$ \\
\hline \multirow[t]{2}{*}{ 3-Hexen-1-ol (Z) } & 176 & 0.002 & $\operatorname{Tr}$ & $\operatorname{Tr}$ & $\operatorname{Tr}$ & $\operatorname{Tr}$ & $\operatorname{Tr}$ \\
\hline & 191 & ND & ND & ND & ND & ND & ND \\
\hline \multirow[t]{2}{*}{ 2-Hexen-1-ol (E)- } & 176 & $0.011 \mathrm{a}$ & 0.001 & 0.001 & 0.001 & 0.001 & 0.001 \\
\hline & 191 & $0.005 \mathrm{~b}$ & $\operatorname{Tr}$ & $\operatorname{Tr}$ & $\operatorname{Tr}$ & $\operatorname{Tr}$ & $\operatorname{Tr}$ \\
\hline \multirow[t]{2}{*}{ 1-Hexanol } & 176 & $0.028 \mathrm{a}$ & $1.115 \mathrm{a}$ & $2.754 \mathrm{a}$ & $7.493 \mathrm{a}$ & $7.152 \mathrm{a}$ & $8.329 \mathrm{a}$ \\
\hline & 191 & $0.037 \mathrm{a}$ & $1.530 \mathrm{a}$ & $1.488 \mathrm{a}$ & $1.282 \mathrm{~b}$ & $2.267 \mathrm{~b}$ & $0.645 \mathrm{~b}$ \\
\hline \multirow[t]{2}{*}{ 1-Heptanol } & 176 & $0.003 \mathrm{a}$ & $0.003 \mathrm{a}$ & $0.003 \mathrm{a}$ & $0.004 \mathrm{a}$ & $0.003 \mathrm{a}$ & $0.004 \mathrm{a}$ \\
\hline & 191 & $0.003 \mathrm{a}$ & $0.003 \mathrm{a}$ & $0.002 \mathrm{~b}$ & $0.002 \mathrm{~b}$ & $0.002 \mathrm{~b}$ & $0.002 \mathrm{~b}$ \\
\hline \multirow[t]{2}{*}{2 Etil 1 hexanol } & 176 & $\operatorname{Tr}$ & $\operatorname{Tr}$ & $\operatorname{Tr}$ & $\operatorname{Tr}$ & $\operatorname{Tr}$ & 0.0005 \\
\hline & 191 & $\operatorname{Tr}$ & $\operatorname{Tr}$ & $\operatorname{Tr}$ & $\operatorname{Tr}$ & $\operatorname{Tr}$ & $\operatorname{Tr}$ \\
\hline \multirow[t]{2}{*}{ 1-Octanol } & 176 & 0.0006 & 0.001 & 0.001 & 0.001 & 0.002 & 0.002 \\
\hline & 191 & $\operatorname{Tr}$ & $\operatorname{Tr}$ & $\operatorname{Tr}$ & $\operatorname{Tr}$ & $\operatorname{Tr}$ & $\operatorname{Tr}$ \\
\hline
\end{tabular}

Medias entre fechas de cosecha dentro de un mismo compuesto y seguidas por la misma letra minúscula, no son significativamente diferentes (Tukey, 0.05). $\mathrm{Tr}=$ Trazas, $<0.0005 \mathrm{ppm} ; \mathrm{ND}=$ compuestos no detectados. 
Cuadro 6. Concentración de ésteres volátiles (ppm) en frutos de la manzana 'Red Delicious' cosechados en dos estados de madurez (176 y 191 días después de plena floración, ddpf).

\begin{tabular}{|c|c|c|c|c|c|c|c|}
\hline \multirow{2}{*}{ Ésteres } & \multicolumn{7}{|c|}{ Almacenamiento (días) } \\
\hline & ddpf & 0 (osecha) & 30 & 60 & 90 & 120 & 150 \\
\hline \multirow[t]{2}{*}{ Acetato de etilo } & 176 & $0.004 \mathrm{a}$ & $0.007 \mathrm{a}$ & $0.006 \mathrm{a}$ & $0.006 \mathrm{a}$ & $0.006 \mathrm{a}$ & $0.422 \mathrm{a}$ \\
\hline & 191 & $1.649 \mathrm{~b}$ & $0.006 \mathrm{a}$ & $0.057 \mathrm{a}$ & $0.040 \mathrm{a}$ & $1.496 \mathrm{~b}$ & $3.387 \mathrm{~b}$ \\
\hline \multirow[t]{2}{*}{ Propionato de etilo } & 176 & $\operatorname{Tr}$ & $0.0005 \mathrm{a}$ & $0.0005 \mathrm{a}$ & $0.0009 \mathrm{a}$ & $0.001 \mathrm{a}$ & $0.014 \mathrm{a}$ \\
\hline & 191 & 0.004 & $0.003 \mathrm{a}$ & $0.004 \mathrm{a}$ & $0.001 \mathrm{a}$ & $0.005 \mathrm{~b}$ & $0.004 \mathrm{~b}$ \\
\hline \multirow[t]{2}{*}{ Acetato de propilo } & 176 & $0.003 \mathrm{a}$ & $1.270 \mathrm{a}$ & $0.601 \mathrm{a}$ & $0.607 \mathrm{a}$ & $0.517 \mathrm{a}$ & $0.293 \mathrm{a}$ \\
\hline & 191 & $0.956 \mathrm{~b}$ & $0.715 b$ & $3.505 \mathrm{~b}$ & $0.441 \mathrm{a}$ & $0.180 \mathrm{a}$ & $0.014 \mathrm{a}$ \\
\hline \multirow[t]{2}{*}{ Butanoato de metilo } & 176 & 0.0009 & 0.0007 & 0.001 & 0.001 & $0.002 \mathrm{a}$ & $0.005 \mathrm{a}$ \\
\hline & 191 & $\operatorname{Tr}$ & $\operatorname{Tr}$ & $\operatorname{Tr}$ & $\operatorname{Tr}$ & $0.006 \mathrm{~b}$ & $0.002 \mathrm{~b}$ \\
\hline \multirow[t]{2}{*}{ Acetato de 2 metil propilo } & 176 & $0.009 \mathrm{a}$ & $0.022 \mathrm{a}$ & $0.023 \mathrm{a}$ & $0.023 \mathrm{a}$ & $0.016 \mathrm{a}$ & $0.020 \mathrm{a}$ \\
\hline & 191 & $0.025 \mathrm{~b}$ & $0.016 \mathrm{a}$ & $0.012 \mathrm{a}$ & $0.021 \mathrm{a}$ & $0.014 \mathrm{a}$ & $0.016 \mathrm{a}$ \\
\hline \multirow[t]{2}{*}{ Metil 2 metil butanoato } & 176 & $0.0005 \mathrm{a}$ & $\operatorname{Tr}$ & $\operatorname{Tr}$ & $\operatorname{Tr}$ & $\operatorname{Tr}$ & 0.0006 \\
\hline & 191 & $0.034 \mathrm{~b}$ & $\operatorname{Tr}$ & $\operatorname{Tr}$ & $\operatorname{Tr}$ & $\operatorname{Tr}$ & $\operatorname{Tr}$ \\
\hline \multirow[t]{2}{*}{ Butanoato de etilo } & 176 & $0.001 \mathrm{a}$ & $0.034 \mathrm{a}$ & $0.037 \mathrm{a}$ & $0.032 \mathrm{a}$ & $0.032 \mathrm{a}$ & $0.792 \mathrm{a}$ \\
\hline & 191 & $0.308 \mathrm{~b}$ & $0.195 \mathrm{a}$ & $0.131 \mathrm{a}$ & $0.035 \mathrm{a}$ & $0.549 \mathrm{~b}$ & $0.955 \mathrm{a}$ \\
\hline \multirow[t]{2}{*}{ Propanoato de propilo } & 176 & $0.001 \mathrm{a}$ & $0.027 \mathrm{a}$ & $0.029 \mathrm{a}$ & $0.017 \mathrm{a}$ & $0.010 \mathrm{a}$ & $0.010 \mathrm{a}$ \\
\hline & 191 & $0.031 \mathrm{~b}$ & $0.047 \mathrm{~b}$ & $0.015 \mathrm{~b}$ & $0.012 \mathrm{a}$ & $0.011 \mathrm{a}$ & $\operatorname{Tr}$ \\
\hline \multirow[t]{2}{*}{ Acetato de butilo } & 176 & $0.035 \mathrm{a}$ & $37.982 \mathrm{a}$ & $40.534 \mathrm{a}$ & $42.481 \mathrm{a}$ & $32.394 \mathrm{a}$ & $32.649 \mathrm{a}$ \\
\hline & 191 & $2.515 \mathrm{a}$ & $8.237 \mathrm{~b}$ & $8.641 \mathrm{~b}$ & $8.664 \mathrm{~b}$ & $6.617 \mathrm{~b}$ & $2.266 \mathrm{~b}$ \\
\hline \multirow[t]{2}{*}{ 2-Metil butanoato de etilo } & 176 & $0.001 \mathrm{a}$ & $0.005 \mathrm{a}$ & $0.005 \mathrm{a}$ & $0.004 \mathrm{a}$ & $0.001 \mathrm{a}$ & $0.014 \mathrm{a}$ \\
\hline & 191 & $0.037 \mathrm{~b}$ & $0.006 \mathrm{a}$ & $0.009 \mathrm{a}$ & $0.0005 \mathrm{a}$ & $0.006 \mathrm{a}$ & $0.012 \mathrm{a}$ \\
\hline \multirow{2}{*}{ Acetato de 2 metil butilo } & 176 & $0.193 \mathrm{a}$ & $22.550 \mathrm{a}$ & $8.052 \mathrm{a}$ & $8.236 \mathrm{a}$ & $5.435 \mathrm{a}$ & $5.172 \mathrm{a}$ \\
\hline & 191 & $3.003 \mathrm{a}$ & $4.883 \mathrm{~b}$ & $4.484 \mathrm{~b}$ & $2.634 \mathrm{~b}$ & $3.800 \mathrm{a}$ & $1.907 \mathrm{~b}$ \\
\hline \multirow[t]{2}{*}{ Pentanoato de etilo } & 176 & $0.001 \mathrm{a}$ & $0.069 \mathrm{a}$ & $0.070 \mathrm{a}$ & $0.076 \mathrm{a}$ & $0.049 \mathrm{a}$ & $0.037 \mathrm{a}$ \\
\hline & 191 & $\operatorname{Tr}$ & $0.092 \mathrm{a}$ & $0.058 \mathrm{a}$ & $0.044 \mathrm{a}$ & $0.081 \mathrm{a}$ & $\operatorname{Tr}$ \\
\hline \multirow[t]{2}{*}{ Propanoato de butilo } & 176 & $0.001 \mathrm{a}$ & $0.020 \mathrm{a}$ & $0.023 \mathrm{a}$ & $0.026 \mathrm{a}$ & $0.020 \mathrm{a}$ & $0.017 \mathrm{a}$ \\
\hline & 191 & $0.011 \mathrm{~b}$ & $0.028 \mathrm{~b}$ & $0.018 \mathrm{a}$ & $0.020 \mathrm{a}$ & $0.014 \mathrm{a}$ & $0.004 \mathrm{~b}$ \\
\hline \multirow[t]{2}{*}{ Acetato de pentilo } & 176 & $0.005 \mathrm{a}$ & $0.041 \mathrm{a}$ & $0.044 \mathrm{a}$ & $0.044 \mathrm{a}$ & $0.046 \mathrm{a}$ & $0.034 \mathrm{a}$ \\
\hline & 191 & $0.023 \mathrm{~b}$ & $0.051 \mathrm{~b}$ & $0.038 \mathrm{a}$ & $0.033 \mathrm{~b}$ & $0.042 \mathrm{a}$ & $0.012 \mathrm{~b}$ \\
\hline \multirow[t]{2}{*}{ Acetato de 3 metil buten-1-ol } & 176 & 0.005 & $\operatorname{Tr}$ & $\operatorname{Tr}$ & $\operatorname{Tr}$ & $\operatorname{Tr}$ & $\operatorname{Tr}$ \\
\hline & 191 & $\operatorname{Tr}$ & $\operatorname{Tr}$ & $\operatorname{Tr}$ & $\operatorname{Tr}$ & $\operatorname{Tr}$ & $\operatorname{Tr}$ \\
\hline \multirow[t]{2}{*}{ Butanoato de butilo } & 176 & $0.003 \mathrm{a}$ & $0.034 \mathrm{a}$ & $0.051 \mathrm{a}$ & $0.051 \mathrm{a}$ & $0.075 \mathrm{a}$ & $0.065 \mathrm{a}$ \\
\hline & 191 & $0.007 \mathrm{a}$ & $0.045 \mathrm{~b}$ & $0.046 \mathrm{a}$ & $0.071 \mathrm{~b}$ & $0.091 \mathrm{~b}$ & $0.010 \mathrm{~b}$ \\
\hline \multirow[t]{2}{*}{ Acetato de cis-3-hexenilo } & 176 & 0.0008 & $\operatorname{Tr}$ & $\operatorname{Tr}$ & $\operatorname{Tr}$ & $\operatorname{Tr}$ & $0.0007 \mathrm{a}$ \\
\hline & 191 & $\operatorname{Tr}$ & $\operatorname{Tr}$ & $\operatorname{Tr}$ & $\operatorname{Tr}$ & $\operatorname{Tr}$ & $0.013 \mathrm{~b}$ \\
\hline Acetato de hexilo & 176 & $0.012 \mathrm{a}$ & $1.977 \mathrm{a}$ & $5.924 \mathrm{a}$ & $3.797 \mathrm{a}$ & $3.440 \mathrm{a}$ & $3.112 \mathrm{a}$ \\
\hline & 191 & $0.102 \mathrm{a}$ & $3.938 \mathrm{~b}$ & $6.267 \mathrm{a}$ & $3.708 \mathrm{a}$ & $5.155 \mathrm{~b}$ & $0.154 \mathrm{~b}$ \\
\hline 2-Metil butanoato de butilo & 176 & $0.001 \mathrm{a}$ & $0.010 \mathrm{a}$ & $0.009 \mathrm{a}$ & $0.006 \mathrm{a}$ & $0.005 \mathrm{a}$ & $0.005 \mathrm{a}$ \\
\hline & 191 & $0.004 \mathrm{a}$ & $0.008 \mathrm{a}$ & $0.006 \mathrm{a}$ & $0.004 \mathrm{a}$ & $0.004 \mathrm{a}$ & $0.001 \mathrm{~b}$ \\
\hline Hexanoato de propilo & 176 & $0.002 \mathrm{a}$ & $0.006 \mathrm{a}$ & $0.006 \mathrm{a}$ & $0.003 \mathrm{a}$ & $0.004 \mathrm{a}$ & $0.004 \mathrm{a}$ \\
\hline & 191 & $0.001 \mathrm{a}$ & $0.005 \mathrm{a}$ & $0.005 \mathrm{a}$ & $0.004 \mathrm{a}$ & $0.007 \mathrm{~b}$ & $\operatorname{Tr}$ \\
\hline Propanoato de hexilo & 176 & $0.005 \mathrm{a}$ & $0.003 \mathrm{a}$ & $0.003 \mathrm{a}$ & $0.009 \mathrm{a}$ & $0.005 \mathrm{a}$ & $0.005 \mathrm{a}$ \\
\hline & 191 & $0.001 \mathrm{~b}$ & $0.003 \mathrm{a}$ & $0.003 \mathrm{a}$ & $0.003 \mathrm{~b}$ & $0.002 \mathrm{~b}$ & $0.001 \mathrm{~b}$ \\
\hline Butanoato de hexilo & 176 & 0.0007 & $0.004 \mathrm{a}$ & $0.006 \mathrm{a}$ & $0.007 \mathrm{a}$ & $0.014 \mathrm{a}$ & $0.009 \mathrm{a}$ \\
\hline & 191 & $\operatorname{Tr}$ & $0.005 \mathrm{a}$ & $0.009 \mathrm{a}$ & $0.016 \mathrm{~b}$ & $0.016 \mathrm{a}$ & $0.002 \mathrm{~b}$ \\
\hline Octanoato de etilo & 176 & $0.002 \mathrm{a}$ & 0.007 & 0.001 & 0.003 & 0.001 & 0.001 \\
\hline & 191 & $0.0008 \mathrm{~b}$ & $\operatorname{Tr}$ & $\operatorname{Tr}$ & $\operatorname{Tr}$ & $\operatorname{Tr}$ & $\operatorname{Tr}$ \\
\hline 2-Metil butanoato de hexilo & 176 & 0.0009 & 0.004 & 0.003 & 0.003 & 0.003 & 0.003 \\
\hline & 191 & $\operatorname{Tr}$ & $\operatorname{Tr}$ & $\operatorname{Tr}$ & $\operatorname{Tr}$ & $\operatorname{Tr}$ & $\operatorname{Tr}$ \\
\hline Hexanoato de hexilo & 176 & 0.0007 & 0.002 & 0.002 & $0.002 \mathrm{a}$ & $0.004 \mathrm{a}$ & 0.004 \\
\hline & 191 & $\operatorname{Tr}$ & $\operatorname{Tr}$ & $\operatorname{Tr}$ & $0.003 \mathrm{a}$ & $0.001 \mathrm{~b}$ & $\operatorname{Tr}$ \\
\hline
\end{tabular}


La manzana 'Golden Delicious' cosechada a los $157 \mathrm{ddpf}$ presentó mayor contenido total de compuestos volátiles (suma del contenido de compuestos del tipo alcohol, aldehído, y éster), que la manzana cosechada a los 176 ddpf, por la alta concentración de aldehídos presentes. Durante las etapas tempranas de almacenamiento (30 a $90 \mathrm{~d})$, el contenido total de compuestos volátiles no mostró diferencias significativas en la manzana 'Golden Delicious' de ambas cosechas ( $\mathrm{P} \leq 0.05)$; sin embargo, al comparar grupos funcionales de compuestos volátiles, en la fruta cosechada antes del inicio del climaterio (157 ddpf) se observó que los aldehídos y alcoholes presentaron valores mayores $(\mathrm{P} \leq 0.05)$, mientras que en la cosechada 176 ddpf al inicio del climaterio, los ésteres mostraron concentraciones mayores durante los tres primeros meses de almacenamiento $(\mathrm{P} \leq 0.05)$; posteriormente no se observaron diferencias significativas (Figura 2).

La producción mayor de compuestos volátiles del tipo éster cuantificados durante los primeros tres meses de almacenamiento en la manzana cosechada a $176 \mathrm{ddpf}$, puede tener un impacto altamente positivo, ya que estos compuestos son los más importantes cualitativamente en el sabor de la manzana (Fellman et al., 2000). Por tanto, la cosecha de 'Golden Delicious' efectuada al inicio del climaterio con CIE de 0.9 ppm puede considerarse más adecuada en cuanto al contenido de compuestos volátiles del tipo éster, que la cosecha muy temprana, antes del climaterio, con CIE de 0.003 ppm.

Estos resultados concuerdan con los reportados por Song et al. (1996) quienes analizaron el desarrollo de compuestos volátiles de manzanas cosechada en varios estados de madurez y almacenadas por $45 \mathrm{~d}$ a $20{ }^{\circ} \mathrm{C}$; estos autores encontraron que la manzana cosechada tempranamente $(\mathrm{CIE}=0.009 \mathrm{ppm})$ produjo menor compuestos volátiles que la cosechada en una fecha óptima ( $\mathrm{CIE}=0.2 \mathrm{ppm})$. Vanoli et al. (1995) encontraron resultados similares al cosechar la manzana a los 158,172 y 181 ddpf y luego almacenarla a $18{ }^{\circ} \mathrm{C}$, pues registraron mayor producción de compuestos volátiles durante el almacenamiento en las cosechadas a los 172 ddpf. Fellman et al. (2000) encontraron que la concentración de ésteres en manzana 'Fuji' aumenta conforme avanzaba la maduración del fruto, cuando los valores de CIE al tiempo de cosecha eran menores de 1 ppm.

Los compuestos volátiles de mayor concentración durante el almacenamiento de la manzana 'Golden Delicious' cosechada a ambos estados de madurez fueron: hexanal, 2-hexenal, cis-3hexenal, 1-hexanol, 2-metil 1-butanol y acetato de butilo (Cuadros 1, 2 y 3). La manzana 'Golden Delicious' cosechada antes del inicio del climaterio (157 ddpf) mostró concentraciones mayores $(\mathrm{P} \leq 0.05)$ de 2-hexenal y 1-hexanol durante todo el almacenamiento; de hexanal a los 120 y $150 \mathrm{~d}$; y de cis 3-hexenal al momento de la cosecha. Los aldehidos 2-hexenal y hexanal aportan un sabor intenso a "fruta verde" (Rizzolo et al., 1989), cis 3-hexenal aporta un sabor a "hoja" (Defilippi et al., 2009), mientras que 1-hexanol aporta un sabor a tierra (Rizzolo et al., 1989).

Por su parte, la manzana 'Golden Delicious' cosechada al inicio del climaterio (176 ddpf) mostró concentraciones mayores $(\mathrm{P} \leq 0.05)$ de 1-butanol durante los primeros 90 d de almacenamiento; de 2 metil 1-butanol a los 90, 120, $150 \mathrm{~d}$ de almacenamiento; y de acetato de butilo a los 60, 90 y $120 \mathrm{~d}$ de almacenamiento (Cuadros 1, 2 y 3). El 1-butanol aporta un sabor "dulce" (Young et al., 1996); 2 metil 1-butanol proporciona un sabor "frutal placentero" (Dimick y Hoskin, 1983), mientras que el acetato de butilo proporciona un sabor a "manzana" (Young et al., 1996).

Los resultados aquí obtenidos muestran que la manzana cosechada tempranamente, antes del inicio del climaterio (157 ddpf), presenta sabores a "manzana verde" durante el almacenamiento, mientras la manzana cosechada al inicio del climaterio (176 ddpf) presenta sabores más característicos de una manzana madura. Sin embargo, es importante señalar que la manzana cosechada a los 176 ddpf tiene una disminución considerable de ésteres después del tercer mes de almacenamiento, lo cual permite indicar que esta fecha de cosecha sólo es conveniente para manzanas que serán almacenadas un máximo de 3 meses; para almacenar por un periodo más prolongado sería más conveniente la primera fecha de cosecha (157 ddpf; Figura 2).

Los compuestos volátiles que se encontraron en mayor concentración en la manzana 'Red Delicious' al momento de la cosecha fueron los aldehídos, después los alcoholes y en menor concentración los ésteres (Figura 3). Durante el almacenamiento de la manzana 'Red Delicious' se observó una disminución considerable en la concentración de aldehídos y un incremento en la concentración de alcoholes y de ésteres. De acuerdo con Sanz et al. (1997), en la síntesis de volátiles, primero se producen los aldehídos (manzana "verde"), y a partir de éstos se producen los alcoholes y de estos ultimos los ésteres (manzana "madura"). La manzana 'Red Delicious' cosechada a los 176 ddpf presentó menor contenido total de compuestos volátiles que la manzana cosechada a los 191 ddpf al momento de la cosecha; sin embargo, durante el almacenamiento la manzana cosechada a los 176 ddpf mostró una mayor concentración $(\mathrm{P} \leq 0.05)$ de compuestos volátiles en comparación con la manzana cosechada a los $191 \mathrm{ddpf}$. Vanoli et al. (1995) cosecharon en manzana 'Starkspur Golden' cosechada a los 158, 172 y 181 ddpf y almacenada a $18{ }^{\circ} \mathrm{C}$, Vanoli et al. (1995) observaron 


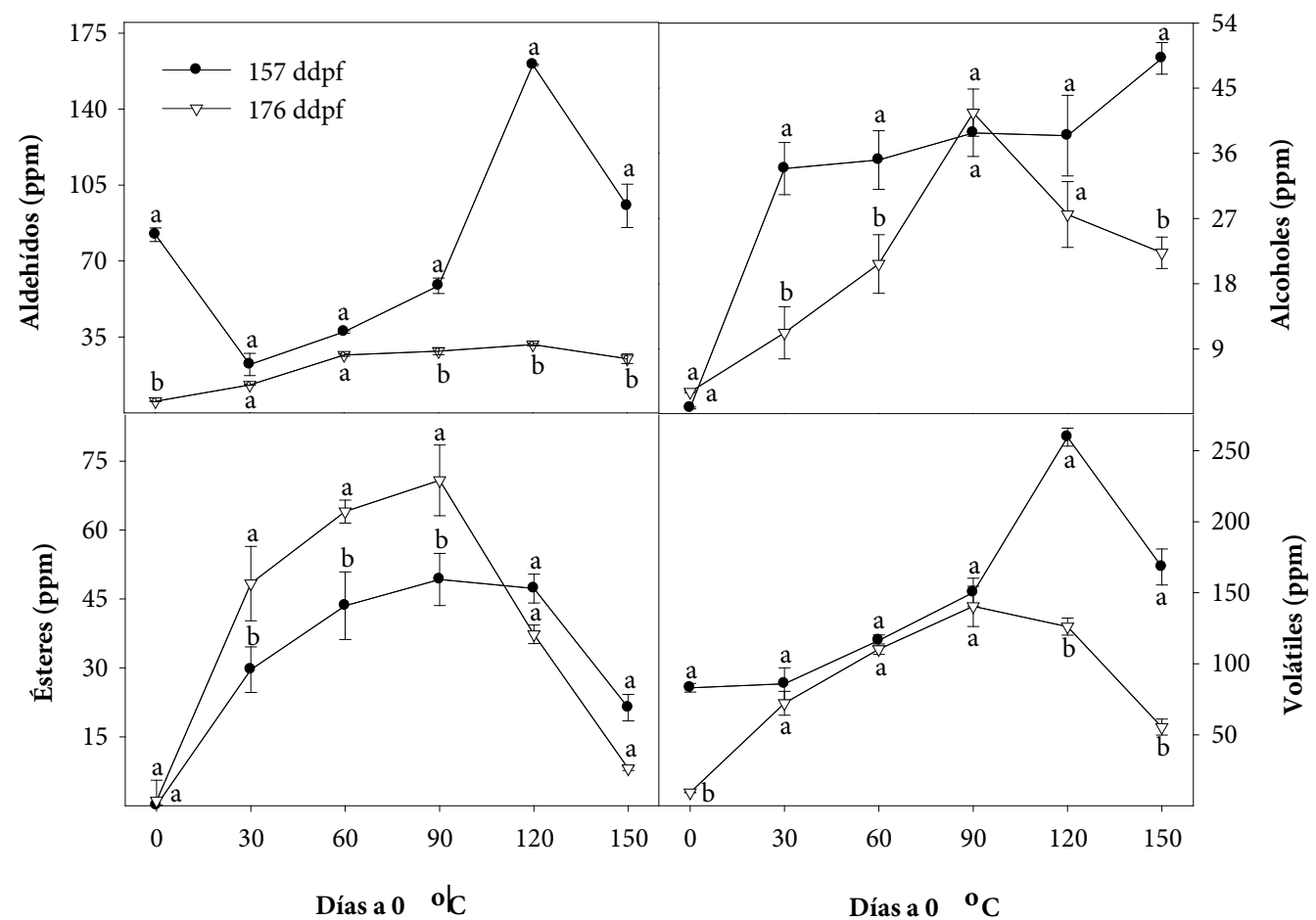

Figura 2. Producción de compuestos volátiles de manzana 'Golden Delicious' cosechadas en dos fechas (157 y 171 ddpf) y almacenada en refrigeración. Medias dentro de un mismo día a $0^{\circ} \mathrm{C}$ seguidas por la misma letra minúscula, indican que las fechas no son significativamente diferentes (Tukey, 0.05).

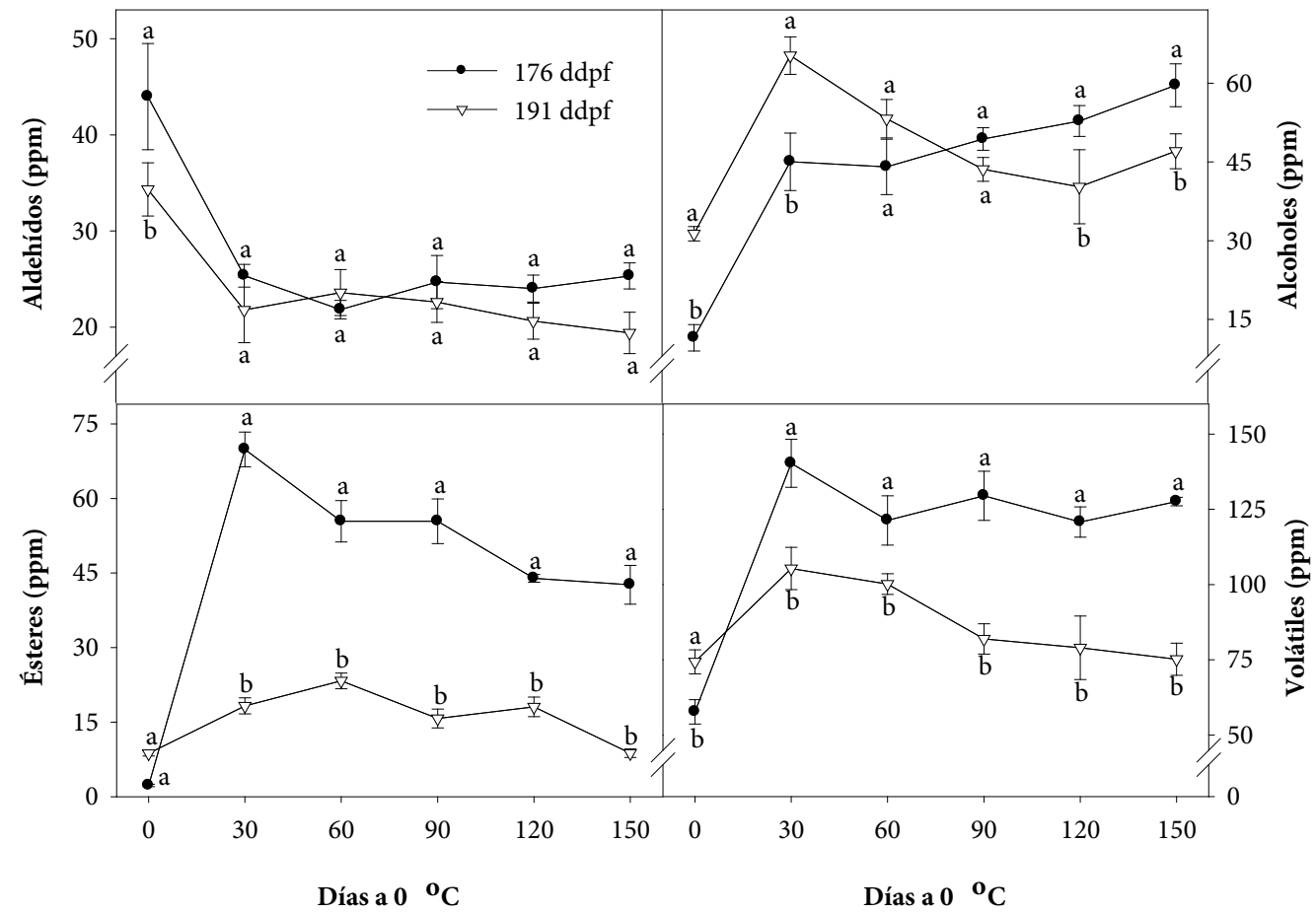

Figura 3. Producción de compuestos volátiles de manzana 'Red Delicious' cosechadas en dos fechas (176 y 191 ddpf) y almacenada en refrigeración. Medias dentro de un mismo día a $0^{\circ} \mathrm{C}$ seguidas por la misma letra minúscula, indican que las fechas no son significativamente diferentes (Tukey, 0.05 ). 
una mayor producción de compuestos volátiles durante el almacenamiento en la manzana cosechada a los $172 \mathrm{ddpf}$.

Song et al. (1996) descubrieron que la fecha óptima de cosecha de la manzana se presenta cuando la CIE exhibe un valor de $0.2 \mathrm{ppm}$, que es cuando el fruto produce más compuestos volátiles durante el almacenamiento a $20{ }^{\circ} \mathrm{C}$ en comparación con manzanas cosechadas con un CIE de $27.1 \mathrm{ppm}$. Los resultados del estudio concuerdan con los reportados por Song et al. (1996) ya que para el desarrollo de compuestos volátiles en frutos bajo almacenamiento refrigerado es más conveniente la cosecha con CIE de 0.3 ppm, que la cosecha con CIE de 17 ppm.

La concentración de aldehídos en 'Red Delicious' no presentó diferencias significativas $(\mathrm{P} \leq 0.05)$ durante el almacenamiento, entre la manzana cosechada al inicio del climaterio (176 ddpf) y en el climaterio (191 ddpf). Sin embargo, la manzana cosechada a los 176 ddpf presentó mayor cantidad de ésteres durante el almacenamiento, en comparación con la manzana cosechada a los $191 \mathrm{ddpf}$. Este comportamiento sugiere que la cosecha de manzana 'Red Delicious' a los 176 ddpf es más adecuada para la acumulación de ésteres, principales compuestos volátiles del sabor (Figura 3).

Los compuestos volátiles de mayor concentración durante el almacenamiento de la manzana 'Red Delicious' fueron: 2-metil 1-butanol, acetato de butilo, 2-hexenal, cis3hexenal, 2 metil butil acetato, y 1-hexanol (Cuadros 4, 5 y 6). La manzana 'Red Delicious' cosechada a los $176 \mathrm{ddpf}$ mostró concentraciones mayores $(\mathrm{P} \leq 0.05)$ de los aldehídos cis 3-hexenal y 2 -hexenal durante el almacenamiento y en la cosecha, compuestos que otorgan el sabor a "inmaduro". El compuesto 2-hexenal es de impacto en la manzanas 'Delicious' (Flath et al., 1967).

La manzana 'Red Delicious' cosechada al inicio del climaterio también presentó concentraciones más altas ( $\mathrm{P}$ $\leq 0.05$ ) de acetato de butilo y acetato de 2-metil butilo, que la manzana cosechada en el climaterio; estos compuestos son de alto impacto en el sabor de la manzana (Kays y Paull, 2004). Según Fellman et al. (2000), los compuestos volátiles del tipo ésteres de acetato, como el acetato de butilo y el acetato de 2-metil butilo son lo que imparten características de sabor dulce, afrutado a la fruta. Los resultados aquí obtenidos sugieren que esta manzana cosechada al inicio del climaterio resulta ser la más apropiada en términos de producción de compuestos volátiles durante el almacenamiento, en comparación con la cosechada en el climaterio.

\section{CONCLUSIONES}

La fecha de cosecha es un factor crítico en el desarrollo del sabor de la manzana durante el almacenamiento. Si las manzanas son cosechadas tempranamente (manzana 'Golden Delicious' cosechada a los 157 ddpf) no desarrollarán su sabor apropiadamente; si las manzanas son cosechadas tardíamente (manzanas 'Red Delicious' cosechadas a los $191 \mathrm{ddpf}$ ) producirán menos volátiles que en la cosecha óptima. Estos resultados evidencian el importante papel del estado de madurez fisiológica de la fruta de manzana al momento de la cosecha por que impacta la producción de compuestos volátiles del sabor durante el almacenamiento, así como la importancia de elegir el momento adecuado de cosecha para optimizar la calidad postcosecha de la manzana.

\section{AGRADECIMIENTOS}

Al Consejo Nacional de Ciencia y Tecnología (CONACyT) y al Gobierno del Estado de Chihuahua a través de los proyectos FOMIX CHIH-2008-C01-92083, y FOMIX CHIH-2010-C01-146966, por aportar los recursos financieros para el desarrollo de esta investigación, y a la Compañía Grupo La Norteñita S.A., por proporcionar los frutos.

\section{BIBLIOGRAFÍA}

Baldwin E A (2002) Fruit flavor, volatile metabolism, and consumer perceptions. In: Fruit Quality and its Biological Basis. M Knee (ed). CRC Press, Boca Raton, FL. pp:89-106.

Beaulieu J C, E A Baldwin (2002) Flavor and aroma of fresh-cut fruits and vegetables. In: O Lamikanra (ed). Fresh-cut Fruit and Vegetables Science, Technology and Market. CRC Press, Boca Raton, FL., USA pp:391-425.

Bismark, Banco de Investigación y Marketing (2002) Manzano: Las Variedades de Más Interés. Irta, Barcelona, España.

Cunningham D G, T E Acree, J Barnard, R Butts, P Braell (1986) Charm analysis of apple volatiles. Food Chem. 19:137-147.

Defilippi B G, D Manríquez, K Luengwilai, M González-Agüero (2009) Aroma volatiles: biosynthesis and mechanisms of modulation during fruit ripening. Adv. Bot. Res. 50:1-37

De Pooter H L, M Van Acker, N M Schamp (1987) Aldehyde metabolism and the aroma quality of stored Golden Delicious apples. Phytochemistry 26:89-92.

Dimick P S, J C Hoskin (1983) Review of apple flavour-state of the art. Crit. Rev. Food Sci. Nutr. 18:387-409.

Fellman J K, T W Miller, D S Mattinson, J P Mattheis (2000) Factors that influence biosynthesis of volatile flavor compounds in apple fruits. HortScience 35:1026-1033.

Flath R A, D R Black, D G Guadagni, W H McFadden, T H Schultz (1967) Identification and organoleptic evaluation of compounds in Delicious apple essence. J. Agric. Food Chem. 15:29-35.

Forney C F, J P Mattheis, A E Baldwin (2009) Effects on Flavor. In: Modified and Controlled Atmospheres for the Storage, Transportation, and Packaging of Horticultural Commodities. E M Yahia (ed). CRC. Boca R, USA. pp:119-159.

Kays S J, R E Paull (2004) Postharvest Biology. Exon Press. Athens, GA. $568 \mathrm{p}$.

Mattheis J P, J K Fellman, P M Chen, M E Patterson (1991) Changes in headspace volatiles during physiological development of Bisbee Delicious apple fruit. J. Agric. Food Chem. 39:1902-1906.

Pérez A G, C Sanz (2008) Formation of Fruit Flavour. In: Fruit and 
Vegetable Flavour. B Bruckner, W S Grant (eds). Cambridge, England. pp:41-70

Rizzolo A, A Polesello, G Y Teleky-Vamossy (1989) CGC/Sensory analysis of volatile compounds developed from ripening apple fruit. J. High Resol. Chromat. 12:824-827.

SAS Institute (2010) Statistical Analysis System Version 8 for Windows Inc. Cary, NC, USA.

SIAP, Servicio de Información Agroalimentaria y Pesquera (2009) Secretaría de Agricultura, Ganadería, Desarrollo Rural, Pesca y Alimentación (SAGARPA). Anuario Estadístico de la Producción Agrícola. Disponible en http://www.siap.gob.mx. (Noviembre 2009).

Salas-Salazar N A, G I Olivas (2011) El aroma de la manzana. Interciencia 36:265-271

Saltveit M (1982) Procedures for extracting and analyzing internal gas samples from plant tissues by gas chromatography. HortScience 17:878-881.

Sanz C, J M Olias, A G Pérez (1997) Aroma biochemistry of fruits and vegetables. In: Phytochemistry of Fruit and Vegetables. F A
Tomas-Barberan, R J Robins (eds). Oxford University, New York. pp:125-155.

Song J, F Bangerth (1996) The effect of harvest date on aroma compound production from 'Golden Delicious' apple fruit and relationship to respiration and ethylene production. Postharv. Biol. Technol. 8:259-269.

Vanoli M, C Visai, A Rizzolo (1995) The influence of harvest date on the volatile composition of Starkspur Golden apples. Postharv. Biol. Technol. 6:225-234.

Wyllie S G (2008) Flavour quality of fruit and vegetables: are we on the brink of major advances? In: Fruit and Vegetable Flavour. B Bruckner, W S Grant (eds). Cambridge, England. pp:3-9.

Wyllie S G, J K Fellman (2000) Formation of volatile branched chain esters in bananas (Musa sapientum L.). J. Agric. Food Chem. 48:3493-3496.

Yahia E M (1994) Apple flavor. Hort. Rev. 16:197-234.

Young H, J M Gilbert, S H Murray, R D Ball (1996) Causal effects of aroma compounds on Royal Gala apple flavours. J. Agric. Food Chem. 71:329-336. 